\section{Von einfachen Merksätzen bis zu wichtigen Leitsymptomen}

— Vielen Ärzten, die auch durchaus langjährig in der Pädiatrie tätig sind, sträuben sich beim Thema "Stoffwechselerkrankungen" die Nackenhaare, da es sich hier für etliche noch immer um ein Buch mit sieben Siegeln handelt. Prof. Dr. Hansjosef Böhles macht es geschickt. Er gewinnt den Leser mit interessanten historischen Themen. So beschreibt er die Leistungen zahlreicher Forscher wie Max Rubner, der in einem Bombenkalorimeter die Energiefreisetzung bei der Verbrennung von Nahrungsbestandteilen bestimmte, oder von Adolf Kußmaul, der bereits 1874 die nach ihm benannte "Kußmaul-Atmung" beim diabetischen Koma publizierte.

Das Buch ist zweigeteilt. Die erste Hälfte befasst sich mit Grundlagenwissen. Wohltuend sind so einfache Merksätze wie „Die Leber ist ein Fettsäuren-konsumierendes und ein Glukose-produzierendes Organ". Für die tägliche Arbeit wichtig sind die Ausführungen zum Natriumhaushalt, zur
Bedeutung des Laktats oder etwa zur Glykogenspeicherkapazität der fetalen Leber. Ob es in einem "Stoffwechselbuch" eigene Propädeutikkapitel zum Verständnis von bildgebenden Verfahren oder von elektrophysiologischen Untersuchungsmethoden bedarf, mag dahingestellt bleiben. Das Kapitel zum metabolischen Labor ist hingegen essenziell. Geruchshinweise oder typische Urinverfärbungen können hilfreiche Markereigenschaften in der frühen Differenzialdiagnose darstellen. Denken wir nur an den Mäusegeruch bei der PKU.

Großartig und wichtig ist insbesondere für den niedergelassenen Praktiker oder den Kliniker eines Krankenhauses an der Basis die zweite Hälfte des Buches, die sich mit den metabolischen Erkrankungen konkret beschäftigt. Die Ausführungen zu den Leitsymptomen - teilweise verdeutlicht mit Patientenfotos - sind sehr hilfreich. Quervernetzte Merksätze wie „Furosemid führt zu Veränderungen wie beim Bartter-Syn- drom" tuen gut. Aber auch seltene Krankheitsbilder wie der Morbus Wolman werden verständlich charakterisiert. Bei der nächsten Auflage sollte die inzwischen verfügbare und seit 2015 in Deutschland zugelassene Enzymersatztherapie mit Sebelipase alfa für diesen lysomalen sauren Lipase-Mangel allerdings Erwähnung finden.

Wertvoll für das differenzialdiagnostische Denken sind die diagnostischen Strategien gegen Ende des Buches. Als Fazit bleiben 500 Seiten komprimiertes Wissen - verfasst von nur einem Autor und somit aus einem Guss - ansprechend und empfehlenswert aufgearbeitet. Das Werk ist zusätzlich mit einer elektronischen Buchversion versehen.

Dr. Thomas Hoppen

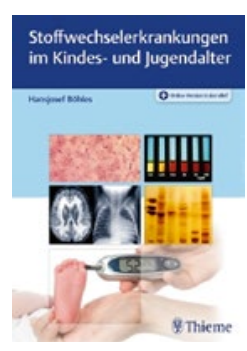

Hansjosef Böhles

Stoffwechselerkrankungen im Kindes- und Jugendalter

Thieme, Stuttgart 2016

ISBN: 9783132007215 $129,99 €$

\title{
Ausgezeichneter sozialpädiatrischer Leitfaden
}

— Der Begriff "Frühe Hilfen" wurde in den letzten Jahren im Zusammenhang mit Prävention und Kinderschutz neu definiert: Sie bilden lokale und regionale Unterstützungssysteme mit Hilfsangeboten für Eltern und Kinder. Der Schwerpunkt liegt in der Altersgruppe von 0 bis 3 Jahren. Zusätzlich wollen die frühen Hilfen Entwicklungsmöglichkeiten von Kindern und Eltern frühzeitig und nachhaltig verbessern, insbesondere bei der Beziehungs- und Erziehungskompetenz von Müttern und Vätern. Damit tragen sie maßgeblich zum gesunden Aufwachsen von Kindern bei und sichern ihre Rechte auf Schutz, Förderung und Teilhabe.

Dieses Ziel, nämlich die Entwicklung von Kindern nachhaltig zu fördern, ist auch ein zentrales Anliegen der Kinderheilkunde, insbesondere der Sozialpädiatrie. In dem vorliegenden Buch werden die Berührungspunkte und Gemeinsamkeiten aller im Gesundheitswesen, im Kinderschutz und der Frühförderung tätigen Fachleute aus Medizin und Sozialarbeit übersichtlich dargestellt. Für die Kinder- und Jugendärzte the- matisiert dieser Leitfaden praxisrelevante Fragen für die tägliche Arbeit, vor allem auch wie ein Bedarf an frühen Hilfen erkannt und welche Angebote den betroffene Familien vermittelt werden können.

In insgesamt 15 Kapitel werden die folgenden Themen behandelt: frühe Hilfen und interventioneller Kinderschutz; Bedarf an frühen Hilfen (Epidemiologie); Belastungsfaktoren und Störungen der frühkindlichen Entwicklung; psychosoziale Belastungen und protektive Faktoren; Screeninginstrumente im Bereich der frühen Hilfen; Strategien der Erkennung im Rahmen der pädiatrischen Früherkennungsuntersuchungen; Schreibabyambulanz, stationäre Sozialpädiatrie und interaktionsorientierte MutterKind-Psychotherapie; Angebote der Kinderund Jugendhilfe, interdisziplinäre Frühförderung und Bedeutung der Schwangerschaftsberatung jeweils im Rahmen früher Hilfen; der Pädiatrische Anhaltsbogen sowie weitere ausgewählte Instrumente für die klinische Praxis zur Einschätzung von psychosozialem Unterstützungsbedarf.
Die Herausgeber und Autoren sind ausgewiesene Fachleute aus den Bereichen Sozialpädiatrie, Kinder- und Jugendpsychiatrie, Sozialwissenschaften und Sonderpädagogik. Nach den rund 280 Seiten verfügt der Leser über solide Kenntnisse im Bereich frühe Hilfen. Das Buch ist sorgfältig lektoriert. Abbildungen, Tabellen, Literatur und Zitate sind so, wie man es von einem führenden medizinischen Verlag erwartet. Für die kinderärztliche Praxis gibt das Buch kluge Denkhilfen, praktikable Lösungsansätze und insgesamt einen ausgezeichneten sozialpädiatrischen Leitfaden an die Hand - das Ganze zu einem vernünftigen Preis.

Dr. Ulrich Mutschler

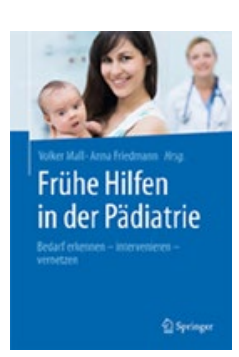

Volker Mall, Anna Friedmann (Hrsg.)

Frühe Hilfen in der Pädiatrie

Springer-Verlag, Berlin Heidelberg 2016

ISBN 978-3-662-49261-1 $49,99 €$

auch als eBook erhältlich $39,99 €$ 